\title{
Uniform vs. Non-Uniform Scaling of Shooter Games on Large Displays
}

\author{
Robert J. Teather, Jacques Carette, Manivanna Thevathasan \\ Dept. of Computing \& Software \\ McMaster University \\ Hamilton, ON, Canada \\ \{teather $\mid$ carette\}@mcmaster
}

\begin{abstract}
We present a study comparing player performance in a shooter game using two different types of scaling across four display sizes. The first scaling type used uniform scaling where increasing the display size also increased the size of all in-game elements by the same factor. The second employed non-uniform scaling where all in-game elements remained fixed in size, but the game environment increased (or decreased) in size. As expected, gameplay becomes much easier at larger scales with non-uniform scaling. Our results quantify this expectation: different difficulty attributes are very well modeled using either linear or power models. We discuss the implications this has on maintaining constant game difficulty and user experience.
\end{abstract}

Keywords - Scaling; shooter games; display size

\section{INTRODUCTION}

Modern display technology presents gamers with a wider range of game platforms than ever before. TV screens have grown ever larger, with high resolutions at affordable prices. At the same time, mobile devices come in a wide range of sizes from very small smartphones to large tablets. And gamers continue to use mid-sized displays such as desktop monitors.

The wide range of display sizes has increased complexity in game development. Mobile developers, in particular, are well aware of scale issues when porting a game from one device to another. Android games, for example, are developed for up to four display sizes. The Android Developer's Guide [1] [1]recommends preparing game assets for each of these sizes. The closest "fit" to the device size is used. For devices between these sizes, content is scaled; all game assets are uniformly stretched by the same factor [1]. Microsoft offers similar recommendations for mobile [2] and desktop applications [3].

Different devices can have the same resolution, yet a different form factor. In this case, scale is handled implicitly and automatically, but can potentially yield unappealing results. On the other hand, developing four sets of assets is expensive. Triple-A studios may choose another route, and develop ultra-high resolution assets and then scale these down. We choose to explore a completely different part of the design space: rather than scaling everything, we fix game elements at their native size but change the size of the game play area. Naturally, this would have a significant effect on difficulty and gameplay, but hopefully in such a predictable manner that we can easily compensate for it. Reasoning that object tracking and path-planning (or steering) tasks are strongly affected by display space, we chose shooter games as a genre where player performance would most likely be strongly affected.

Our objective is to explore ways of normalizing user experience across different display sizes. We expect that nonuniform scaling will yield an inconsistent user experience, but this inconsistency might be addressed through automated means. For example, increasing the number of enemies or their speed as a function of the display size might yield equivalent user experience. We thus also model player performance based on display size, models which will be instrumental in normalizing the user experience across different display sizes.

\section{BACKGROUND}

Display size has been studied in certain UI tasks, including document navigation [4], navigating virtual environments [5, 6], spatial orientation [7], pointing or point selection tasks [811], steering (path following) [12] and pursuit tracking (moving target acquisition) [13].

While the results of these studies favour larger displays, scale effects may be task dependent. Bridgeman et al. [14] report no difference across display size in a quantity comparison task using graphical visualizations. But larger displays do improve reading tasks [14]. Others report that larger displays elicited positive subjective responses and may improve the sense of presence with moving video, but not for still images [15]. This likely also applies to dynamic games.

Games involve substantially different tasks than many UI problems, often requiring constant attention and fast reactions from the player. However, there is little work directly evaluating scale effects in games [16]. Moreover, the game porting process is often handled in an ad-hoc fashion [17]. Developers often must rely on experience and instinct, rather than empirically founded suggestions for scaling. Our work addresses this via empirical experimentation.

Since there is relatively little work on games and scale, we look to similar tasks. Virtual reality (VR), like games, uses a tightly-coupled feedback loop requiring constant attention from the user and constant input to control the system effectively. Thus we speculate that we may find similar results as those in studied of scale in VR $[6,7,18]$, even though the game we developed does not use a 3D view.

Ni et al [5] studied large and small displays across high and low resolutions in a VR navigation task. They report that both size and resolution significantly affected performance, with 


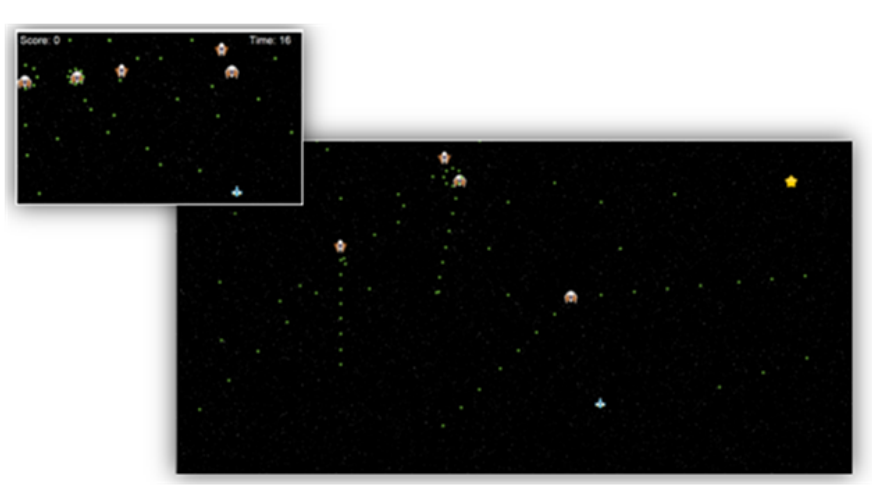

(a)

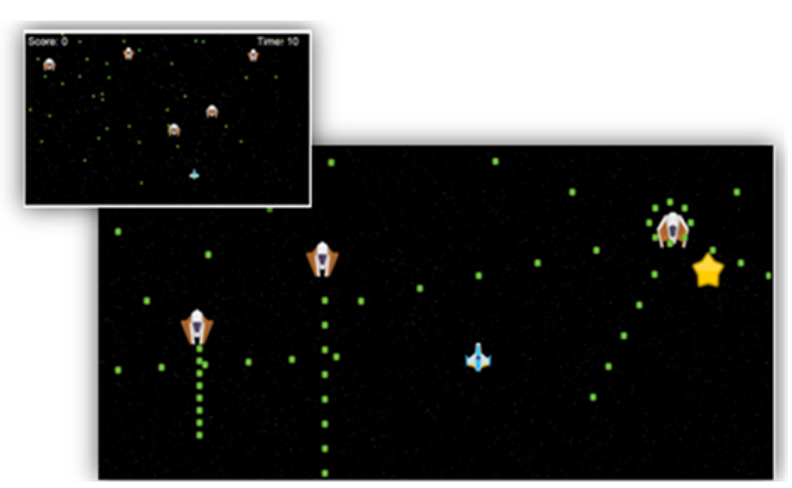

(b)

Fig. 1. The two scale types used in our study. (a) Non-uniform scaling at a large and small scale. Note that in-game elements (player and enemy ships, bullets, etc.) stay consistently sized while the game play space becomes larger. (b) Uniform scaling at large and small scale. In this condition, all ingame elements become larger with larger scales (or smaller at small scales).

higher resolution and larger display size yielding significantly faster task completion. Note that they effectively compared two different visual angles. Tan et al [6] conducted a similar study, but maintained constant visual angle. That is, the large display was positioned such that it occupied the same space on the retina as the small display. Despite the fact that the two displays would appear to be the same size, they report that the larger display offered better performance, allowing participants to more accurately follow a path in the virtual environment.

Responsive web design also delivers special versions of websites designed for specific resolutions [19]. The designer must create the website for each resolution and the browser uses CSS3 media queries to determine which to present.

\section{UNIFORM AND NON-UNIFORM SCALING}

Our study employs two scale types, which we refer to as uniform and non-uniform scaling, see Figure 1. Note that this figure shows extreme examples to make the difference between them more evident.

\section{1) Uniform Scaling Implementation}

Uniform scaling is a "standard" scaling; game elements uniformly scale to match the size of the display. This is done automatically across displays with different sizes but the same resolution. It can also be accomplished by stretching game elements. For example, if one display is twice as large as another, doubling the number of pixels of each game element (in both directions) will uniformly scale them (see Fig. 1b).

To uniformly scale an element in our game, we first compute the default aspect ratio, $D A R=W / H$ where $W$ and $H$ are the default width and height of the asset (in pixels) respectively. We then calculate scale factors width ratio (WR) and height ratio (HR) for both width and height of the element.

$$
\begin{aligned}
& W R=\frac{\text { target_width }}{\text { native_width }} * D A R \\
& H R=\frac{\text { target_height }}{\text { native_height }} * D A R
\end{aligned}
$$

where target_width and target_height represent the target resolution (in pixels) of the display being scaled to, while native_width and native_height are the original resolution the assets were designed for.

\section{2) Non-Uniform Scaling Implementation}

With non-uniform scaling, each game asset can scale differently. In our experiment, we use this to scale the game play area while fixing the size of all in-game elements. Thus the player and enemy ships, bullets and text remain fixed in size, and the game world changes size (see Fig. 1a). In this case, $W R$ and $H R$ are determined by:

$$
W R=H R=\frac{\text { target_width }}{\text { target_height }} * D A R
$$

\section{3) Asset Size}

Ultimately, regardless of which scaling mode is employed the size of game assets as determined by

$$
\begin{gathered}
W^{\prime}=\text { constant } * W R \\
H^{\prime}=\text { constant } * H R
\end{gathered}
$$

where $W^{\prime}$ and $H^{\prime}$ are the newly scaled asset width and height respectively (in pixels). A specific constant is also used for each asset. This allows scaling assets with respect to each other (independent of scaling type.

\section{B. Applications of Non-Uniform Scaling}

The primary intent of non-uniform scaling is explore a different means of adapting to different screen sizes other than naïve scaling, while still keeping the game difficulty constant. Note that this may not apply equally well to all games, and may drastically affect user experience depending on the game genre. It may thus necessitate other changes to normalize experience across different scale levels. We speculate on the impact of non-uniform scaling on specific genres below.

\section{1) Overhead-View Shooter Games}

We studied non-uniform scaling with a space shooter "bullet hell" style game, largely because these games involve tracking large numbers of moving objects and steering tasks. We suspect that such tasks would show pronounced differences in player performance under different kinds of scaling. Here, the space around the player can grow larger on bigger displays. However, this has the effect that the player has proportionally longer to plan in a genre that is designed to overwhelm them with enemies and projectiles from multiple directions. Consequently, we argue that instead the number of enemies, 
projectiles, and their speed can be increased with display size to help maintain a constant level of difficulty.

\section{2) Side-Scrolling Platformer Games}

Non-uniform scaling in platformer games would increase the viewable space, allowing the player to see farther ahead. Similar approaches are used in multiplayer platform games (e.g., Nintendo's New Super Mario Bros. U) to keep all players are on-screen at once. However, these games reduce in-game elements in size in order to show a correspondingly larger playing field (and hence keep all players on-screen). Our approach yields this effect while still maintaining proportionally larger characters (Fig. 2). Of course, for single player games, this may eliminate some of the challenge by giving the player extra time to plan jumps, and presenting enemies earlier than they would otherwise be seen. If part of the games' challenge fundamentally requires a restricted world-view, then non-uniform scaling should not be used.

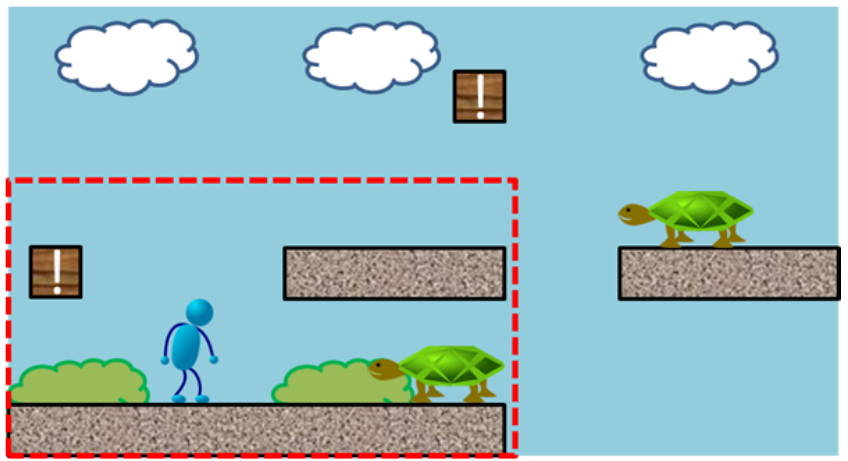

Fig. 2. A (hypothetical) platformer game subject to non-uniform scaling. The dashed box represents the smaller display size, while the full figure represents the larger display size.

\section{3) First-Person Shooter Games}

FPS games effectively already employ non-uniform scaling by way of offering field-of-view control. Using a larger display with non-uniform scaling is equivalent to increasing the field of view - more of the game environment becomes visible (Fig. 3 ). Unlike the game genres mentioned here, no adjustments are necessary to ensure consistent experience. In other words, for many FPS, developers have already decided that the size of the field of view does not significantly change the user experience.

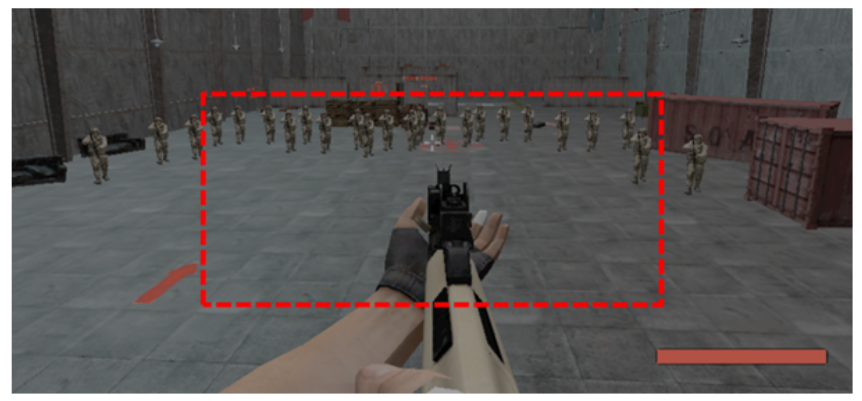

Fig. 3. A FPS game using non-uniform scaling (equivalent to field-of-view). Dashed box shows the smaller display size.

\section{4) Real-Time Strategy Games}

Strategy games offer similar considerations as shooter games. By increasing the play area size, players would more easily see incoming waves of enemies. While this would clearly impact the strategic nature of the game, developers might choose to increase unit movement speed proportional to the increased display size. This should yield a more consistent experience at different levels of scale.

\section{ISSUES IN StUdying SCALE}

Here we discuss several issues in experiments involving scale which motivate our design decisions.

\section{A. Visual Scale: Resolution, Size, and Pixel Density}

Evaluating the effects of scale is complicated due to the interrelationship of several factors. Notably, these include the display size, resolution, and pixel density. Display size refers to the physical size of the display, typically measured on the diagonal and in physical units such as inches. Resolution is number of pixels in the horizontal and vertical dimensions, while pixel density refers to how many pixels are present within a physical unit (often measured in pixels per inch):

$$
\text { Pixel Density }=\frac{\text { Screen Resolution }}{\text { Physical Display Size }}
$$

As implied by (6), if one factors is held constant, then the other two will change together. Note that resolution is commonly fixed - consider the range of 1080p HDTV sizes. Our experiment varies screen resolution as it is the most straightforward method to simulate display resizing via software. To this end, we use a single large HDTV screen, and adjust resolution to simulate a 24 in., 37 in., 50 in. and 75 in. (diagonal) display. Because the viewing distance was approximately $15 \mathrm{ft}$. (from viewer to screen) we argue that the pixel density is less important: individual pixels are not visible at this distance. There is also evidence that the perceived target size on the retina is most important in perceptual scale experiments $[9,20]$. Furthermore, all other potential confounding variables, such as display latency, colour differences, brightness, pixel response time, etc. are all controlled by using a single display.

Finally, note that viewing distance is also a concern, as perspective also influences perceived scale [9]. However, changing viewing distance with scale conditions yields yet another confound in the design, as it becomes unclear whether perspective scaling or software scaling is responsible for measured differences in conditions. Consequently we use a fixed viewing distance to avoid the issue. We plan to revisit viewing distance in future work.

\section{B. Scaling of Non-Visual Game Properties}

In addition to the size of gameplay elements, numerous other aspects of the game change with scale. These factors may directly influence player performance and experience while playing. In the context of shooter games, this includes parameters such as the apparent speed of the player ship, enemy ships, and projectiles.

Note that usually all of these parameters are fixed. Speed, for example, is expressed as pixels moved per second. With uniform scaling, as the number of pixels increase (i.e., on larger display sizes) this yields apparently slower motion. However, all elements appear to move proportionally slower 
by the same factor. On non-uniform scaling, motion speed appears to be constant. A major question of our work is which of these provides a more consistent user experience. A topic for future work is to determine how best to adjust these factors to improve consistency between scale levels.

This may also influence the number of projectiles and enemies present on the screen at once, especially with nonuniform scaling. In our experiment, all enemy spawn rates and bullet firing rates are fixed. On a small non-uniformly scaled display size, this yields a higher density of enemies and projectiles, making the game harder. Conversely, on a large non-uniformly scaled display size, the density of enemies and projectiles decreases, yielding an easier game.

Another issue that influences game experience is task focus. We chose a shooter game since despite the simplistic nature of the game, there are several different valid user strategies. Players could, for example focus on shooting enemies which decreases the number of obstacles and projectiles on the screen at once. Alternatively, players could focus on avoiding enemies and projectiles altogether.

We suspect that scale will influence which strategy works best. For example, on a large scale (especially non-uniform) it becomes harder to take in enough information about incoming enemies/projectiles that are still far away, and more difficult to shoot such enemies. Hence, it may be more fruitful to focus on avoiding nearby obstacles instead. In this case, the avoidance strategy is favoured. Conversely, on smaller scales, it may be easier to plan shots to hit distant enemies, making the shooting strategy more viable.

\section{Methodology}

We present an experiment comparing uniform and nonuniform scaling at several scale factors.

\section{A. Participants}

Sixteen participants (12 male) took part in the study. Their mean age was 21.6 years (SD 3.5 years). Results of a postexperimental demographic survey indicated that all had normal or corrected to normal vision, and only three were left-handed. Fig. 4 presents a summary of participant game experience.

\section{B. Apparatus}

\section{1) Software Setup}

We developed a custom game as our experimental platform. Although we are also interested in evaluating commercial games, these increase experimental complexity [21], and hence will be investigated in future work.

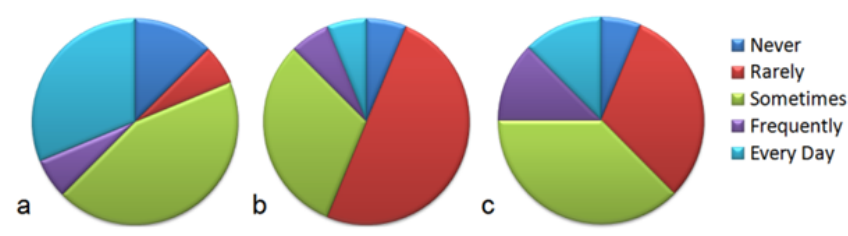

Fig. 4. Participant game playing experience for each of (a) computer games played with mouse/keyboard, (b) console games played with a game controller, and (c) mobile games played with a tablet or smartphone.

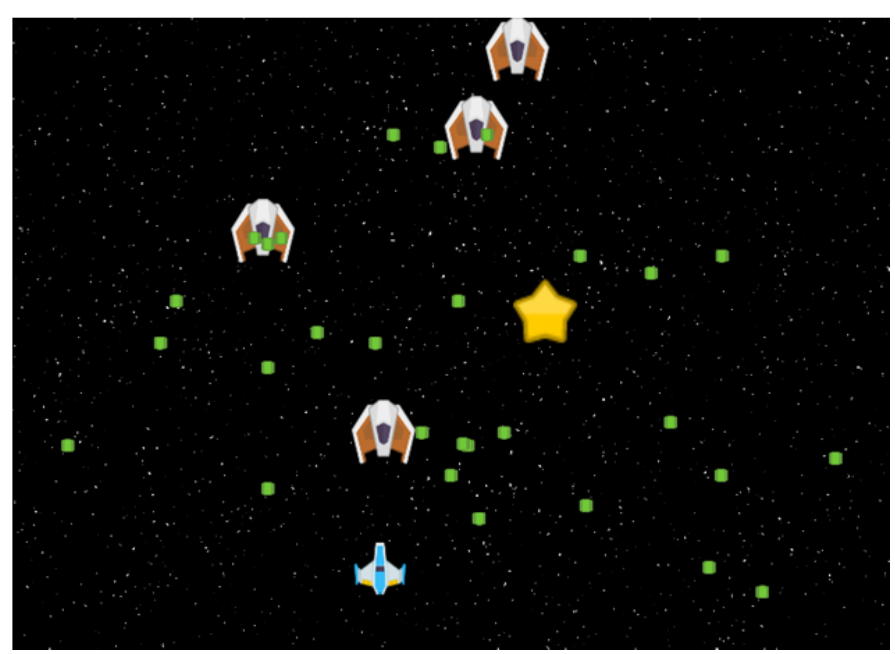

Fig. 5. The game used in the experiment. The player ship is shown in blue and white at the bottom-centre of the image. Multiple enemy ships are shown (orange/gray). The small green squares are enemy bullets the player must avoid. The star increases the player's score.

Software was developed in HTML 5 and CSS 3 using the open-source game engine CraftyJS (http://craftyjs.com/), based on Javascript. We ran the game in the Google Chrome web browser, in full-screen mode. The game ran locally to avoid network delay issues.

The software presented a space-shooter based on arcade style "bullet hell" games (Fig. 5). The game was played by avoiding enemies and their bullets. Two kinds of enemies appeared (randomly). One fired bullets in a straight line downward toward the bottom of the screen. The other fired five bullets simultaneously (two sideways and three forward in a cone) that spread outward as they moved. Hitting an enemy or a bullet destroyed the player, and counted as a player hit. In each one minute trial, six bonus stars also appeared, that increased the player's score. The player ship could also shoot. Successfully shooting an enemy ship destroyed it. Audio feedback was used when shooting (a laser sound effect) and when the player ship was destroyed (an explosion sound effect).

The software scaled the in-game contents to fit specified resolutions. The game contents were sized such that the "nominal" display size (37 in. diagonal) was 945 x 532 pixels, which we refer to as "medium" scale. The scale factors are shown in TABLE I.

In TABLE I. , physical units indicate the corresponding (simulated diagonal) display size. These were chosen as representative of widely available TV sizes. Pixels dimensions varied with physical units, since as discussed earlier, these two factors cannot be decoupled on the same display. View distance was held constant at $15 \mathrm{ft}$. This distance was chosen because it yielded a viewing angle of $2.5 \mathrm{x}$ the display diagonal for the maximum scale factor, which is often cited as an optimal viewing distance ${ }^{1}$. The diagonal measurement (in both pixels and physical units) is given for reference as some results are stated in terms of the diagonal.

${ }^{1}$ See Wikipedia article on HDTV viewing distances for details (http://en.wikipedia.org/wiki/Optimum_HDTV_viewing_distance) 
TABLE I. SCALE FACTORS USED IN THE EXPERIMENT.

\begin{tabular}{|c|c|c|c|}
\hline $\begin{array}{c}\text { Scale Factor } \\
\text { (physical size) }\end{array}$ & $\begin{array}{c}\text { Width } \\
\text { (pixels) }\end{array}$ & $\begin{array}{c}\text { Height } \\
\text { (pixels) }\end{array}$ & $\begin{array}{c}\text { Diagonal } \\
\text { (pixels) }\end{array}$ \\
\hline Small (24 in.) & 614 & 346 & 705 \\
\hline Medium (37 in.) & 945 & 532 & 1085 \\
\hline Large (50 in.) & 1280 & 720 & 1469 \\
\hline Full (75 in.) & 1920 & 1080 & 2203 \\
\hline
\end{tabular}

The software logged several performance metrics, notably, how often the player ship was hit by enemies/bullets, the average time they stayed alive in a session, game score, enemies destroyed, and stars collected. We used the Javascript SDK from Parse ${ }^{2}$ for this, which offers online logging.

\section{2) Hardware Setup}

The experiment was conducted on a $3.4 \mathrm{GHz} \mathrm{PC}$ with a quad-core Intel Core i7 processor, $8 \mathrm{~GB}$ of RAM, and running Windows 7. A 75 in. Samsung Series 77100 Smart TV (1920 x 1080 pixel resolution) was used for all scale conditions. The display was set to run in game mode to minimize input latency.

The game was played with a Nintendo Wii U Pro Controller. See Fig. 6 (inset). The controller was connected to the PC using a USB Bluetooth radio. Controller input was mapped to key events using the WiiUProControllerWin software ${ }^{3}$. The software mapped the A button to shoot, and the start button to progress trials. The left analog stick moved the player ship in the corresponding direction.

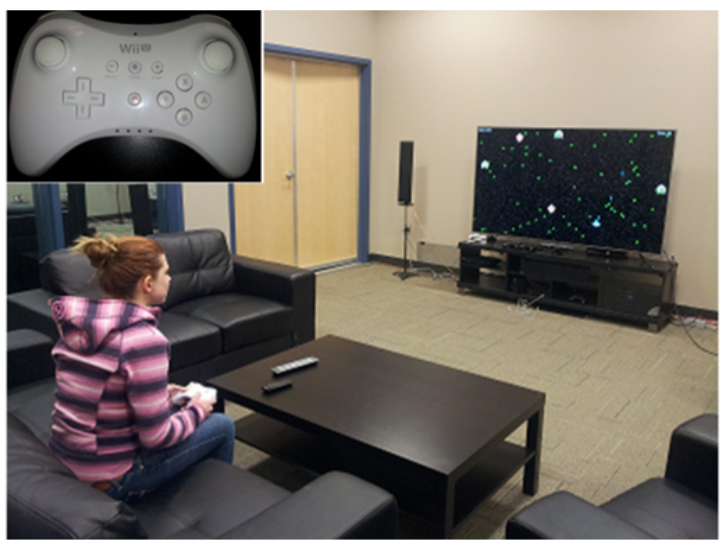

Fig. 6. Lab setup showing the relative position of the couch and TV, while a participant plays the shooter game. Inset: The Wii-U Pro Controller used in the study.

\section{Procedure}

Participants first gave written informed consent. The experimenter then explained the purpose of the experiment and demonstrated the game at the medium scale level. This was intended to show participants the nominal play conditions, and the game objectives. Following this introduction, participants were seated in a mock living-room setup. They sat on a couch positioned at $15 \mathrm{ft}$. from the TV. See Fig. 6.

\footnotetext{
${ }^{2}$ https://parse.com

${ }^{3}$ Software by TeHaxor69 available (April 7, 2015) from

https://docs.google.com/file/d/0B6QxbmaAgfWLZWJ6WFpHYXNjdTA
}

Participants were instructed to play the game to the best of their ability. They were not instructed on particular strategies i.e., they were free to avoid or shoot enemies at their discretion. Each trial lasted one minute, after which a summary screen presented participants with performance metrics. They were prompted to press start to progress, and thus could take breaks between trials.

\section{Design}

We used a $2 \times 4 \times 5$ within-subjects design. The independent variables and their levels were:

Scale Type: uniform, non-uniform

Scale Factor: small, medium, large, full

Trial: 1, 2, 3, 4, 5

TABLE I. summarizes the actual size of each scale factor. The eight combinations of scale type and scale factor were counterbalanced by a balanced Latin square. Trial progressed sequentially and each trial was 1 minute long.

Participants completed six one minute trials for each condition. However, the first trial in each condition was a nonrecorded practice trial, leaving five recorded trials for each condition. In total, each participant thus completed $2 \times 4 \times 5=$ 40 recorded trials, or 640 trials in total over all 16 participants. With introduction and the post-experiment survey, it took each participant approximately 1 hour to complete the experiment.

The dependent variables include player hits (count), longest life duration (in seconds), and enemy kill ratio (percentage).

\section{E. Results}

Results for each dependent variable were analyzed with repeated measures ANOVA, and summarized in Fig. 7 through 9 . To better visualize this data, we adjusted the figure $x$-axes according to the diagonal size of each simulated display scale. This diagonal measure is depicted along the bottom axis of each figure. The corresponding scale factors are shown along the top axis. This transformation effectively normalizes the relative distance between the four scale factor conditions according to their diagonal display size measurement. Ultimately, this makes the trends in the data more apparent than simply plotting them by each scale factor. Results for each dependent variable are discussed in the following sub-sections.

\section{1) Longest Life Duration}

Longest life duration was the longest time between the start of a trial and the player being struck by an enemy or bullet, i.e., the maximum time they were alive for in any given trial, averaged over all trials for each condition. This metric gives an indication of how well participants were able to avoid enemies and survive longer in the game.

The main effect for scale type on longest life duration was significant $\left(F_{1,15}=27.2, p<.001\right)$, as was the main effect for scale factor $\left(F_{3,15}=228.6, p<.0001\right)$. Their interaction effect was also significant $\left(F_{3,15}=31.2, p<.0001\right)$. The group effect was not significant, suggesting that counterbalancing was effective $\left(F_{7,15}=1.8, p=.22\right)$. Trial was not significant $\left(F_{4,15}=1.8, \quad p=.14\right)$. This indicates that participant performance did not improve much over the course of testing. Average scores for longest life duration are seen in Fig. 7. 


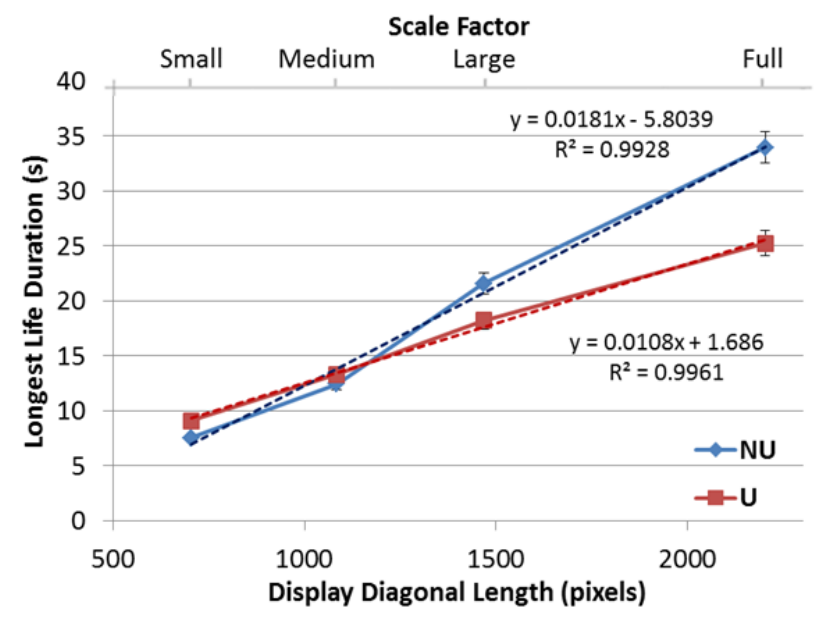

Fig. 7. Longest life duration by scale factor and scale type. NU is nonuniform scaling and $U$ is uniform scaling. Error bars show $\pm 1 S E$. Dashed lines show linear regression model for each scale type. Higher scores are better.

Modeling a dependent variable as a function of an independent variable helps glean a better understanding of control-display relationships in a user interface [22]. In this case, we have modeled each of our dependent variables as a function of scale (using the display diagonal). The dashed lines in Fig. 7 show the linear model of scale factor for each scale type condition. Interestingly, these are nearly perfectly linear, with correlation coefficients approaching 1 . The models for each scale type are also shown in the figure.

\section{2) Enemy Kill Ratio}

Enemy kill ratio was the number of enemies destroyed by the player divided by the number of enemies spawned in a given trial. This was averaged over all trials for each condition. This metric indicates how "aggressive" a participant's play style was, and how effective they were at destroying the enemies. The main effect for scale factor on enemy kill ratio was significant $\left(F_{3,15}=92.2, p<.0001\right)$, but the main effect for scale type was not $\left(F_{1,15}=1.8, p=.22\right)$. The interaction effect, however, was significant $\left(F_{3,15}=18.2, p<.0001\right)$. Trial was not significant $\left(F_{4,15}=1.4, p=.25\right)$. Once again, the group effect was also not significant $\left(F_{7,15}=1.4, p=.34\right)$. See Fig. 8 .

According to Scheffe's posthoc test at the 5\% level, the uniform-small condition had significantly lower enemy kill ratios than any other condition. The non-uniform small, medium, and large conditions and uniform-medium and large were not significantly different. Non-uniform full was significantly higher than the rest of these, while uniform-full offered the highest enemy kill ratios. Like longest life duration, enemy kill ratio increases linearly with scale size. Again, the correlations are extremely high $(>0.98)$ for both scale types.

\section{3) Player Hits}

Player hits was the number of times the player was struck either by an enemy or bullet during a trial, averaged over all trials for a condition. This indicates how successful participants were at avoiding enemies and bullets.

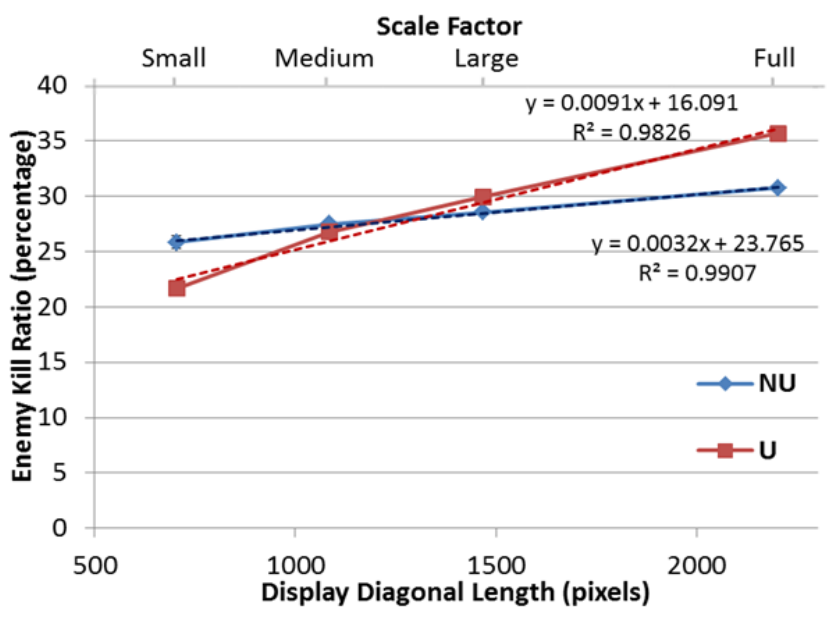

Fig. 8. Average enemy kill ratio for each condition. NU is non-uniform scaling and $\mathrm{U}$ is uniform scaling. Error bars show $\pm 1 S E$. Dashed lines show linear regression model for each scale type. Higher scores are better.

The main effect for scale type on player hits was not significant $\left(F_{1,15}=0.91, \mathrm{~ns}\right)$. The main effect for scale factor, however, was significant $\left(F_{3,15}=200.9, \quad p<.0001\right)$. The interaction effect between scale type and scale factor was also significant $\left(F_{3,15}=27.2, p<.0001\right)$. The group effect was not significant $\left(F_{7,15}=0.97, \mathrm{~ns}\right)$ suggesting that counterbalancing was also effective for this dependent variable. Finally, the main effect for trial approached significance $\left(F_{4,15}=2.7, p=.051\right)$. Mean player hits for each condition are shown in Fig. 9.

The statistical groupings for player hits are largely evident in Fig. 9. Individual conditions were compared using Scheffé's posthoc test at the $5 \%$ level. Non-uniform and uniform scale were significantly different at small scale and full scale, but not at the medium and large scales. As with longest life duration and enemy kill ratio, we attempted to model player hits as a function of the display's diagonal measure. Unlike the other two dependent variables, player hits cannot be approximated by a linear model. Instead, a power model fits the data best.

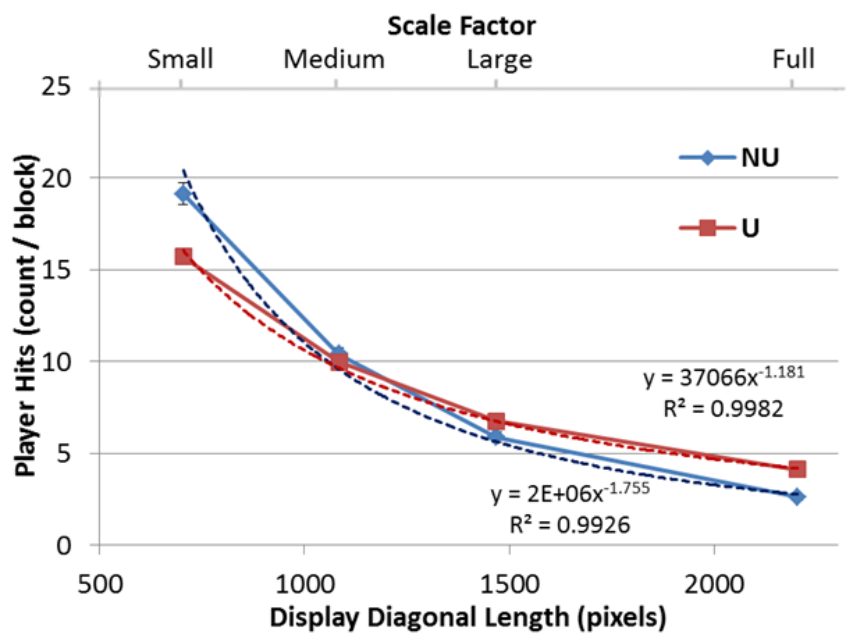

Fig. 9. Average player hits per trial for each condition. NU is non-uniform scaling and $U$ is uniform scaling. Error bars show $\pm 1 S E$. Dashed lines show power model for each scale type. Lower scores are better. 


\section{F. Participant Preferences}

The post-experiment survey included questions regarding general participant preference for the conditions. The first set of such questions asked participants about the perceived difficulty of a given condition relative to medium scale. For example, participants were asked With uniform scaling, and relative to medium scale, how did the difficulty change with a larger scale? Similar questions were asked for non-uniform and smaller scale sizes. Results of this set of questions are summarized in Fig. 10, which depicts proportions of each response relative to neutral (no difference). Clearly participants perceived a strong difference between the conditions, favouring larger scale factors, especially with NU scaling.

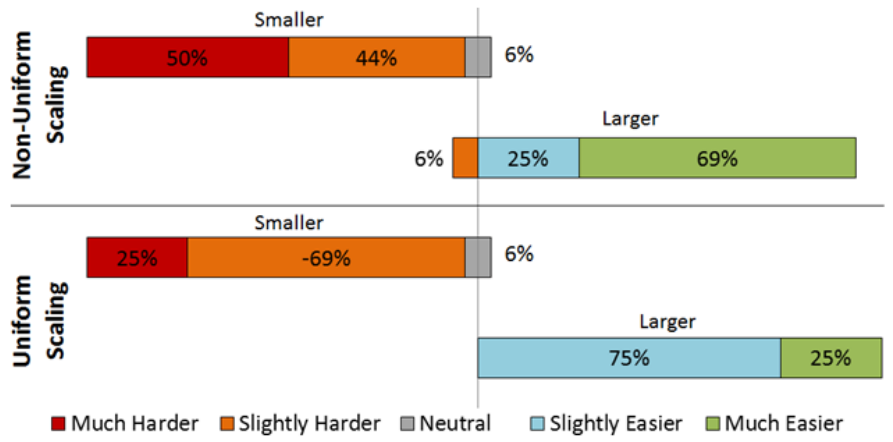

Fig. 10. Perceived difficulty of conditions relative to medium scale, by condition. From top to bottom, NU scaling with smaller scale factors, then larger scale factors. Then, U scaling with smaller scale factors, then larger scale factors.

\section{DISCUSSION}

\section{A. Scaling Type}

It is clear that non-uniform scaling made the game significantly harder at large scale, and significantly easier at small scale - as expected. The participants were also aware of this. One noted that "the small displays were very challenging as there was not a lot of space to maneuver or plan ahead for dodging". The participant further remarked that non-uniform scaling with small scale "was particularly hard because the objects on the screen were so big compared to the available space".

Based on the reported performance differences, a side effect of NU scaling is that player experience changes drastically with scale. However, a surprising result is how predictably performance varies as a function of size - either linear or as a power curve (depending on which metric of performance). Hence, we argue that adjusting for this factor should be rather straightforward! For example, one could consider maintaining consistent game difficulty by adjusting enemy spawn rates, movement speeds, firing rates, and so on. This may yield a more consistent experience, but is a topic for future study.

\section{B. Scale Factor}

Scale factor also significantly affects participants' ability to play the game. In general, across both uniform and nonuniform scaling, larger scale factors improved performance. This is consistent with previous results $[5,9,13,18]$. The effect of scale factor in non-uniform scaling is naturally more pronounced than with uniform. This should make it easier to modify factors such as enemy speeds, firing rates, etc., depending on scale, to counter the change in difficulty.

Note that for all dependent variables, significant interaction effects were revealed between scaling type and scale factor. In general, the medium and large scale factors were not significantly different between the two scaling types. This was not the case for small and full scale factors, however. These scale factors were consistently significantly different for all dependent variables across scaling type.

The interaction effects described above have a cross-over point for all dependent variables that is between medium and large scale factors. The original game contents were developed for a size slightly larger than medium. It is interesting, though perhaps unsurprising, that this cross-over point reveals the scale factor (slightly larger than medium) where one would expect uniform and non-uniform scaling to perform exactly the same. Effectively, this is the scale factor where the two are indistinguishable. Phrased differently, this is the "ideal" size of the in-game elements.

\section{Predictive Models}

We produced predictive models for all dependent variables as a function of screen size. The models for longest life duration and enemy kill ratio were surprisingly linear for both scaling types. The model for player hits best fit a power curve.

A key motivation of our work is to explore different ways of adapting games to different sized display, beyond naïve scaling. The models presented in our results indicate that performance changes over scale factor according in highly predictable ways. Since the fit of the models was so high (approaching $R^{2}$ of 1.0), we take this as a promising sign that it will be feasible to provide consistent player experience when using non-uniform scaling.

The models form a basis with which to programmatically normalize experience over scale factor. Future work will investigate this issue further. It is currently unclear, for example, if a linear increase (with scale factor) of the enemy spawn rate would compensate for additional space offered at larger non-uniform display scales.

\section{Limitations and Future Work}

The main limitation of this work is that we tested our scaling techniques on only a single game genre. Clearly the effectiveness of the scaling method is at least partly dependent on the type of game being played. For certain genres of games, we expect that the utility of non-uniform scaling could range from somewhat ineffective to wholly inappropriate. For puzzle games (e.g., Tetris), this type of scaling is likely not applicable; expanding the play space would fundamentally change the nature of the game. Note that this may be why the port of Plants vs Zombies on the Xbox 360 does not use the full play area, but instead expands the size of the scenery to fill the space.

On the other hand, certain game genres might benefit. As mentioned earlier, side-scrolling (2D) platformer games are one genre we are interested in testing further. Existing games (e.g., Nintendo's New Super Mario Bros. U) already use a form 
of scaling for multiplayer games - when players move far apart, the game world scales up in size to keep multiple players on-screen at once. A downside to this is that game content decreases in size, which can yield harder-to-see enemies and obstacles. Our approach to non-uniform scaling might work similarly should a larger screen be available, without the limitation of decreasing in-game content size.

The second main limitation of this work is that we used a fixed view distance. While this is clearly somewhat unrealistic (e.g., players are unlikely to play on a 24 " screen at the same distances as a 75 " screen) we argue that this was the correct choice from an experimental methodology point of view. The alternative of moving players to a different "preferred" distance for each display size would introduce a confounding effect: Two forms of scaling (perspective and artificial/software scaling) would be simultaneously applied. Hence another avenue for future work is to assess the effects of viewing distance. By moving the viewer, different scale factors can be made to appear visually consistent - e.g., by ensuring that all scale factors conform to the same visual angle. It is unclear if normalizing scale across viewing distance in this fashion will influence performance, or if it would elicit further differences between uniform and non-uniform scaling. Future work will thus investigate if moving to a "preferred" distance for each simulated display size improves results.

A final issue is that the player experience is inconsistent across different scale levels. That main open question is how to change other game properties (e.g., enemy spawn rates, firing rates, speed, etc.) such that players perceive they are playing "the same" game across multiple scale factors. The models presented above represent our first attempt at understanding this "normalization" process.

\section{CONCLUSIONS}

We presented an empirical comparison of uniform and nonuniform scaling. As expected, non-uniform scaling yielded a significantly easier game experience at larger levels of scale, and significantly harder at smaller scales. Interestingly, most of our dependent variables were extremely well modeled as functions of the display size for both uniform and non-uniform scaling.

Although we conducted our study using a large display system, we suspect similar results would be found using a small displays (e.g., mobile devices). We argue that nonuniform scaling should be considered as one more method to adapt games to different screen sizes. Future work will focus on additional methods and thus offer viable design alternatives for certain game genres.

\section{REFERENCES}

[1] A. Developers. (2010, May 7). The Android Developer's Guide: Supporting Multiple Screens. Available: https://developer.android.com/ guide/practices/screens_support.html. Accessed Aug. 27, 2015.

[2] Microsoft. (2014, May 17). Supporting multiple resolutions in Windows 8.1 apps. Available: http://msdn.microsoft.com/en-us/library/windows/ apps/dn263244.aspx. Accessed Aug. 27, 2015.

[3] R. Haveson and K. Sykes. (2008, May 17). Writing DPI-aware Win32 applications. Available: http://download.microsoft.com/download/ 1/f/e/1fe476f5-2b7a-4af1-a0ed-
768454a0b5b1/Writing\%20DPI\%20Aware\%20Applications.pdf. Accessed Aug. 27, 2015.

[4] M. R. Jakobsen and K. Hornbæk, "Sizing up visualizations: Effects of display size in focus+context, overview+detail, and zooming interfaces," Proceedings of the ACM SIGCHI Conference on Human Factors in Computing Systems - CHI 2011, 2011, pp. 1451-1460.

[5] T. Ni, D. A. Bowman, and J. Chen, "Increased display size and resolution improve task performance in information-rich virtual environments," in Proceedings of Graphics Interface 2006, 2006, pp. 139-146.

[6] D. S. Tan, D. Gergle, P. G. Scupelli, and R. Pausch, "Physically large displays improve path integration in 3D virtual navigation tasks," in Proceedings of the SIGCHI Conference on Human Factors in Computing Systems - CHI 2004, 2004, pp. 439-446.

[7] D. S. Tan, D. Gergle, P. Scupelli, and R. Pausch, "With similar visual angles, larger displays improve spatial performance," Proceedings of the SIGCHI Conference on Human Factors in Computing Systems - CHI 2003, 2003, pp. 217-224.

[8] O. Chapuis and P. Dragicevic, "Effects of motor scale, visual scale, and quantization on small target acquisition difficulty," ACM Transactions on Computer-Human Interaction, vol. 18, pp. 1-32, 2011.

[9] J. P. Hourcade and N. Bullock-Rest, "How small can you go? Analyzing the effect of visual angle in pointing tasks," Proceedings of the ACM SIGCHI Conference on Human Factors in Computing Systems - CHI 2012, 2012, pp. 213-216.

[10] Y. Wang, C. Yu, Y. Qin, D. Li, and Y. Shi, "Exploring the effect of display size on pointing performance," Proceedings of the ACM International Conference on Interactive Tabletops and Surfaces - ITS 2013, 2013, pp. 389-392.

[11] A. Kovacs, J. Buchanan, and C. Shea, "Perceptual influences on Fitts' law," Experimental Brain Research, vol. 190, pp. 99-103, 2008.

[12] J. Accot and S. Zhai, "Scale effects in steering law tasks," Proceedings of the ACM SIGCHI Conference on Human Factors in Computing Systems - CHI 2001, 2001, pp. 1-8.

[13] G. Browning and R. J. Teather, "Screen scaling: Effects of screen scale on moving target selection," Extended Abstracts of the ACM SIGCHI Conference on Human Factors in Computing Systems - CHI 2014, 2014, pp. 2053-2058.

[14] B. Bridgeman, M. L. Lennon, and A. Jackenthal, "Effects of screen size, screen resolution, and display rate on computer-based test performance," Applied Measurement in Education, vol. 16, pp. 191-205, 2003.

[15] W. IJsselsteijn, H. de Ridder, J. Freeman, S. E. Avons, and D. Bouwhuis, "Effects of stereoscopic presentation, image motion, and screen size on subjective and objective corroborative measures of presence," Presence: Teleoperators and virtual environments, vol. 10, pp. 298-311, 2001.

[16] R. J. Teather, M. Thevathasan, and J. Carette, "Scale Effects in "Bullet Hell" Games," in IEEE Consumer Electronics Games, Entertainment, and Media - GEM 2014, 2014, pp. 303-304.

[17] V. Alves, I. Cardim, H. Vital, P. Sampaio, A. Damasceno, P. Borba, and G. Ramalho, "Comparative analysis of porting strategies in J2ME games," Proceedings of the IEEE International Conference on Software Maintenance - ICSM 2005, 2005, pp. 123-132.

[18] D. S. Tan, D. Gergle, P. Scupelli, and R. Pausch, "Physically large displays improve performance on spatial tasks," ACM Transactions on Computer-Human Interaction (TOCHI), vol. 13, pp. 71-99, 2006.

[19] B. Frain, Responsive web design with HTM15 and CSS3: Packt Publishing Ltd, 2012.

[20] P. van Donkelaar, "Pointing movements are affected by size-contrast illusions," Experimental Brain Research, vol. 125, pp. 517-520, 1999.

[21] R. P. McMahan, E. D. Ragan, A. Leal, R. J. Beaton, and D. A. Bowman, "Considerations for the use of commercial video games in controlled experiments," Entertainment Computing, vol. 2, pp. 3-9, 2011.

[22] I. S. MacKenzie, "Motor behaviour models for human-computer interaction," in HCI models, theories, and frameworks: Toward a multidisciplinary science, J. M. Carroll, Ed., ed: Morgan Kaufman, 2003, pp. 27-54. 